\title{
Esperança, Medo e Qualidade de vida Relacionada à Saúde na Percepção de Mulheres com Câncer de Mama
}

doi: https://doi.org/10.32635/2176-9745.RBC.2021v67n3.1193

\author{
Hope, Fear, and Health-Related Quality of Life Perceived by Women with Breast Cancer \\ Esperanza, Miedo y Calidad de Vida Relacionada a la Percepción de Mujeres con Cáncer de Mama
}

Leandro Aparecido dos Santos Ribeiro'; Marlon Noronha Araújo²; Tânia Maria da Silva Mendonça

RESUMO

Introdução: O período de diagnóstico e tratamento do câncer de mama é difícil, permeado de incertezas e associado a uma alta taxa de morbimortalidade. Objetivo: Conhecer o significado que as mulheres com câncer de mama em tratamento em um hospital universitário atribuíram à sua experiência de medo mascarado pelo sentimento de esperança, bem como investigar a percepção da qualidade de vida no enfrentamento da doença. Método: Estudo quanti-qualitativo com 78 mulheres. Para a avaliaçáo quantitativa, avaliaram-se os escores do Medical Outcomes Study 36 - Item Short - Form Health Survey (SF-36) e da Escala de Esperança de Herth. Para a análise qualitativa, conduziram-se narrativas com dez dessas mulheres que foram analisadas por meio de métodos fenomenológicos de investigação. Resultados: Foram detectados escores reduzidos nos domínios capacidade funcional e saúde mental do SF-36 que implicam em impacto negativo na qualidade de vida e alto índice nos escores de esperança, o que é um fator positivo. Três classes centrais emergiram de 243 segmentos das narrativas por meio de declaraçóes significativas e unidades de significado correspondentes. Conclusáo: A experiência do período de diagnóstico e tratamento inclui enfrentamento, conhecimento da doença, assistência clínica (médicos, equipes), exaltação ao desespero e o paradoxo entre o medo e a esperança. Foi discutida a essência da experiência vivenciada e compartilhada pelas participantes à luz da teoria da doença, como uma ruptura da narrativa biográfica. $\mathrm{O}$ estudo destaca implicaçóes para a equipe de médicos, coordenadores do Sistema Único de Saúde e outros profissionais da saúde.

Palavras-chave: Neoplasias da Mama/psicologia; Qualidade de Vida; Esperança; Emoçóes; Medo.

\section{ABSTRACT}

Introduction: The period of diagnosis and treatment of breast cancer is tough, full of uncertainties, and associated with a high morbidity and mortality rate. Objective: To understand the meaning that women with breast cancer under treatment in a university hospital attribute to their experience of fear masked by the feeling of hope and investigate the participants' perception of quality of life while coping with the disease. Method: Quantitative, qualitative study conducted with 78 women. For the quantitative evaluation, the scores of the Medical Outcomes Study $36-$ Item Short - Form Health Survey (SF-36) and the Herth Hope Scale were evaluated. For the qualitative analysis, narratives were conducted with 10 of these women who were analyzed using phenomenological investigation methods. Results: Low scores were detected in the functional capacity and mental health domains of SF-36, which imply in a negative impact on the quality of life and a high index of hope scores, which is a positive factor. Three central classes emerged from 243 segments of the participants' narratives, through meaningful statements and their corresponding meaning units. Conclusion: The experience of the diagnosis and treatment period includes coping, cognizance of the disease, clinical consultation (doctors, teams), from exaltation to despair, and the paradox between fear and hope. It was discussed the essence of the experience the participants lived and shared in light of the theory of the disease as a rupture of the biographic narrative. The study highlights implications for the teams of physicians, SUS - National Health System coordinators, and other healthcare professionals.

Key words: Breast Neoplasms/psychology; Quality of Life; Hope; Emotions; Fear.
RESUMEN

Introducción: El período de diagnóstico y tratamiento del cáncer de mama es difícil, puesto que presenta muchas incertidumbres y está asociado con una alta taza de morbilidad. Objetivo: Conocer el significado que las mujeres con cáncer de mama en tratamiento en un hospital universitario le atribuyeron a su experiencia de temor enmascarado por el sentimiento de esperanza, así como investigar la percepción de la calidad de vida al enfrentar la enfermedad. Método: Estudio cuantitativo y cualitativo con 78 mujeres. Para la evaluación cuantitativa, evaluamos las puntuaciones del Medical Outcomes Study 36 - Item Short - Form Health Survey (SF-36) y la Escala de Esperanza de Herth. Para el análisis cualitativo, se realizaron narrativas con 10 de estas mujeres que fueron analizadas utilizando métodos de investigación fenomenológica. Resultados: Se detectó que la capacidad funcional y de salud mental del SF-36 se redujeron, y al mismo tiempo se presentó un alto índice de Esperanza lo que implica un impacto negativo en la calidad de vida y un alto índice de puntajes de esperanza, lo cual es un factor positivo. Surgieron tres clases centrales de entre los 243 segmentos de las entrevistas a través de declaraciones significativas y sus respectivas unidades de significado. Conclusión: El experimento, durante el periodo de diagnóstico y tratamiento, incluye el enfrentamiento, el conocimiento de la enfermedad, la asistencia clínica (médicos, equipos), la exaltación a la desesperación y una paradoja entre el miedo y la esperanza. Se discutió la esencia de la experiencia vivida y compartida por las participantes a la luz de la teoría de la enfermedad, como una ruptura de la narrativa biográfica. El estudio destaca las implicaciones para el equipo de médicos, coordinadores del Sistema Único de Salud de Brasil y otros profesionales de la salud. Palabras clave: Neoplasias de la Mama/psicología; Calidad de Vida; Esperanza; Emociones; Miedo.

\footnotetext{
1-3Universidade Federal de Uberlândia. Faculdade de Medicina. Uberlândia (MG), Brasil.

'E-mail: leandroasribeiro@gmail.com. Orcid iD: https://orcid.org/0000-0002-0417-4329

2E-mail: araujomarlon@gmail.com. Orcid iD: https://orcid.org/0000-0001-7304-4205

3E-mail: taniamsm@ufu.br. Orcid iD: https://orcid.org/0000-0001-8392-672X

Endereço para correspondência: Tânia Maria da Silva Mendonça. Av. Pará 1720, bloco 2U, sala 23, B - Umuarama. Uberlândia (MG), Brasil. CEP: $38405-320$. E-mail: taniamsm@ufu.br
} 


\section{INTRODUÇÃO}

Segundo a Organização Mundial da Saúde (OMS), o câncer é o principal problema de saúde e a segunda maior causa de morte no mundo ${ }^{1}$. Entre as mulheres, o câncer de mama é o mais frequentemente diagnosticado e a principal causa de mortalidade nos países desenvolvidos e em desenvolvimento ${ }^{2}$.

Vários estudos já exploraram aspectos da vida das mulheres com câncer de mama usando questionários padronizados, com foco em suporte social, imagem corporal, qualidade de vida $(\mathrm{QV})$ e reaçóes psicológicas ${ }^{3-5}$. Contudo, falta a associação de um estudo qualitativo para confrontar os achados obtidos por meio de questionários e uma investigação sobre a percepção da QV e da esperança e a forma como o medo da progressão da doença integra a vida das pacientes com câncer de mama.

A experiência do adoecimento gera sentimentos antagônicos no ser humano, tais como o medo da progressão da doença e a esperança da cura ${ }^{6}$. Ambos, em dicotomia, reproduzem no indivíduo inconstâncias em suas perspectivas de vida ${ }^{6}$. Das primeiras manifestaçôes fisiopatológicas no organismo ao diagnóstico, tratamento e acompanhamento, as mulheres com câncer experimentam sentimentos antagônicos de medo e esperança, e isto reflete no seu cotidiano e na forma como elas ressignificam suas perspectivas de vida ${ }^{7}$.

Apesar da tendência de melhora no manejo e tratamento das pacientes, o câncer de mama continua afetando a QV geral e a qualidade de vida relacionada à saúde (QVRS) no diagnóstico, durante o tratamento e após a recuperação ${ }^{8}$. QV é um conceito amplo e multifacetado, que incorpora os domínios físicos, psicossociais e espirituais da vida de um indivíduo ${ }^{8}$. A QVRS reflete até que ponto o bem-estar do indivíduo nessas áreas é afetado por uma doença ou pelo tratamento a ela relacionado ${ }^{8}$.

O medo da progressão da doença, definido como a probabilidade subjetiva de resultados negativos, está associado a fatores biopsicossociais negativos em relação ao indivíduo e às suas percepçôes, principalmente em relação à adaptação às limitações impostas pela doença e ao mau prognóstico ${ }^{9}$. Em contrapartida, a esperança de cura, definida como a probabilidade subjetiva de bons resultados, incrementa significado positivo para o cotidiano dos indivíduos, ultrapassando a negatividade das doenças ameaçadoras da vida e proporcionando bem- estar e adaptaçáo aos fatores de estresse ocasionados pelo adoecimento?.

Considerando-se a saúde como a capacidade de autogestấo e adaptação às mudanças cotidianas que proporciona equilíbrio biopsicossocial, é a esperança que impulsiona o homem a seguir em frente ${ }^{10}$. Ela atua como suporte para diversas possibilidades do futuro, pois nutre a capacidade de sonhar e prosseguir em meio às dificuldades, fortalecendo, assim, a resiliência em relaçáo ao processo de adoecimento e tratamento ${ }^{11-13}$.

Assim, pelo fato de a QVRS, a esperança e o medo constituírem-se como fenômenos de extrema importância para o ser humano, alerta-se para a necessidade de profissionais da saúde incluírem, em sua análise de cuidados, a análise da QVRS e identificarem a dicotomia existente entre o medo de progressão da doença e a esperança. Assim, eles proporcionam maior suporte e melhor qualidade na prestação de serviços aos pacientes e desmistificam essas concepçóes por meio da educação e da promoção da saúde, desenvolvendo, com isso, uma compreensão da relevância individual desses três fenômenos no cotidiano pós-diagnóstico e no tratamento das pacientes com câncer de mama ${ }^{13}$.

O uso da abordagem fenomenológica genuína possibilita alavancar a busca de sentido na vida das pessoas, sem que ela se misture ou se confunda com outros fenômenos ${ }^{14}$. Sendo assim, o objetivo deste estudo foi conhecer o significado que as mulheres com câncer de mama em tratamento em um hospital universitário atribuíram à sua experiência de medo mascarado pelo sentimento de esperança, bem como investigar a percepção da QV no enfrentamento da doença.

\section{MÉTODO}

Estudo transversal e quanti-qualitativo de mulheres com câncer de mama, conduzido no Setor de Oncologia do Hospital de Clínicas da Universidade Federal de Uberlândia (HC/UFU), Uberlândia (MG), Brasil. Após aprovação do Comitê de Ética em Pesquisa dessa instituição (CAAE: 80679417.5.0000.5152/Parecer 2.527.653), foram coletados dados para avaliar o impacto da doença na percepção da QV e no nível de esperança de cura. Utilizou-se a avaliação qualitativa, a fim de identificar o significado da doença para esse grupo de mulheres. Foram incluídas pacientes com idade superior a 18 anos, com capacidade cognitiva para entender e responder aos instrumentos de coleta de dados e que concordaram em participar do estudo por meio da assinatura do Termo de Consentimento Livre e Esclarecido (TCLE). Após a assinatura do TCLE, as pacientes que desistiram de participar foram excluídas do estudo.

Para a abordagem quantitativa, os dados foram lançados, a priori, no software G*Power 3.1.9.2, a fim de calcular o tamanho amostral mínimo, considerando o teste de hipóteses para regressão linear múltipla e assumindo tamanho de efeito de 0,10, alfa de 0,05 e poder do teste de 0,80 em uma populaçáo de 256 
mulheres. Nessas condições, a amostra mínima necessária foi de 64 participantes. Acrescentou-se uma margem para possíveis perdas e recusas de 20 pontos percentuais, o que determinou uma amostra final de 78 participantes.

$\mathrm{Na}$ abordagem qualitativa, considerou-se o critério de saturação de dados proposto por Strauss e Corbin ${ }^{15}$, no qual o total de participantes é finalizado assim que os objetivos da investigação são atendidos, assim nenhum novo tema emerge. Esse objetivo foi alcançado assim que as narrativas de dez das participantes incluídas no estudo foram coletadas.

As participantes responderam a um instrumento para coleta de dados sociodemográficos (idade, estado civil, cor, escolaridade, renda familiar e moradia), clínicos e terapêuticos (tempo de diagnóstico, subtipo tumoral, estadiamento clínico, estado menopausal e tipo de quimioterapia). Além do mais, relataram fatores de risco para a doença (idade, história reprodutiva e fatores endócrinos, comportamentais, ambientais, genéticos e hereditários).

Utilizou-se a versão brasileira da Escala de Esperança de Herth (do inglês, Herth Hope Index) ${ }^{16,17}$ para avaliação da esperança. Essa escala possui 12 itens escritos de forma afirmativa, com quatro opçôes de respostas do tipo Likert (discordo, discordo completamente, concordo e concordo completamente). O escore varia de 12 a 48 pontos, sendo que, quanto maior o escore, maior o nível de esperança.

A fim de avaliar a QVRS, utilizou-se o Medical Outcomes Study 36 - Item Short - Form Health Survey (SF-36), versão RAND, validado para o Brasil ${ }^{18}$. O SF-36 apresenta um escore final que varia de 0 a 100, o que corresponde a uma pior e a uma melhor QVRS, respectivamente ${ }^{18}$.

Para avaliar o significado do sentimento das pacientes com relaçáo à doença, dois pesquisadores coletaram as narrativas das participantes. Esses pesquisadores eram acadêmicos do curso de Medicina da Faculdade de Medicina da UFU-MG, que possui um currículo de metodologia inovadora: os estudantes já são inseridos desde o início do curso nos diversos cenários de cuidado dos pacientes, incluindo o setor de oncologia.

Optou-se pela abordagem fenomenológica contemporânea, baseada nos postulados de Husserl ${ }^{19}$, para procurar o significado do período de diagnóstico e o tratamento do câncer de mama. Segundo Husserl ${ }^{19}$, capta-se a essência daquilo que é visto, aquilo que o objeto é em si mesmo, isto é, "ir ao encontro das coisas em si mesmas".

Segundo Creswell ${ }^{20}$, a fenomenologia transcendental contém quatro suposições básicas. $\mathrm{Na}$ primeira, o conhecimento começa com a descrição da experiência, pois busca por sabedoria. Já na segunda, a fenomenologia tenta excluir os julgamentos sobre o que é real, até que se obtenha mais certeza, processo denominado de epoche $e^{20}$. $\mathrm{Na}$ terceira, a intencionalidade da consciência postula que a realidade de um objeto está intimamente ligada à sua consciência e ao significado que se encontra dentro dele ${ }^{20}$. Por fim, na quarta suposição, a fenomenologia exige a recusa da dicotomia sujeito-objeto, e a realidade é apenas no sentido da experiência do indivíduo ${ }^{20}$.

De acordo com Moustakas ${ }^{21}$, no estudo fenomenológico, exploram-se as experiências vividas e relatadas pelos atores, a fim de compreender com precisão a essência invariável de uma experiência. No caso deste estudo, receber o diagnóstico de câncer de mama e passar pelo tratamento tornam-se experiências em si mesmas à medida que as intervençôes acontecem e os resultados não são conhecidos. Mas, como o que os pacientes levam em consideração é a experiência vivida, e não meramente variáveis e escores de um instrumento de avaliaçáo ${ }^{22}$, a fenomenologia foi eleita como a teoria dessa investigação qualitativa.

\section{DADOS QUANTITATIVOS}

O teste de Shapiro Wilk foi utilizado para análise da normalidade das distribuiçôes de dados. Utilizou-se a estatística descritiva para caracterização sociodemográfica, clínica, terapêutica, além dos fatores de risco para o câncer de mama das participantes do estudo.

A qualidade metodológica da avaliação da QV foi verificada por meio do Checklist $\operatorname{COSMIN}^{23}$ e da esperança dos dados mediante análise dos efeitos piso e teto para cada item dos instrumentos utilizados. A confiabilidade dos instrumentos foi testada por meio do coeficiente alfa Cronbach, sendo considerada ideal acima de $0,7^{24}$. Os escores dos domínios do SF-36 foram comparados entre si pelo teste do qui-quadrado.

As médias dos escores da esperança e de QVRS foram comparadas segundo o tempo de diagnóstico em menor do que um ano, de um a três anos e maior do que cinco anos por meio da ANOVA-One Way, com post-hoc de Tukey para identificação de diferenças. O nível de significância considerado foi de $5 \%$.

Os dados foram analisados com o SPSS Statistics (IBM Corp., lançado em 2014 para Windows, versão 24.0, NY, EUA).

\section{DADOS QUALITATIVOS}

A fim de garantir que as participantes se sentissem à vontade para compartilhar suas experiências e não fossem coagidas, as entrevistas começaram com o pedido de que "nos ajudassem a entender suas experiências únicas e se tornassem coinvestigadoras junto conosco"25. A frase disparadora: "Você poderia me contar a história da sua doença?", foi utilizada como protocolo de direcionamento das entrevistas. 
As narrativas foram gravadas e transcritas na íntegra pelos autores. Os nomes das participantes foram alterados, trocando-os por flores, assim como quaisquer informaçóes que pudessem identificá-las, para garantir o seu anonimato.

Os dados foram analisados por meio da hermenêutica-dialética descrita por Minayo ${ }^{26}$. Sendo assim, a trajetória metodológica deste estudo consistiu na gravação e transcrição de cada narrativa, no registro das primeiras impressóes no trabalho em um diário de campo e no resumo dos termos e pontos importantes e recorrentes para facilitar a compreensáo da lógica e dos sentidos das narrativas. Tal prática, denominada hermenêutica, contribuiu para o processo interpretativo, pois foi dinâmico e não fragmentado. $\mathrm{O}$ processo interpretativo ocorreu ao longo do trabalho de campo e manteve um diálogo com os conteúdos que emergiram e com os sentidos que se construíram em cada narrativa. Assim, a lógica do grupo de participantes foi sendo gradativamente apreendida em um movimento parte-todo.

Posteriormente, as narrativas foram analisadas de maneira crítica e reflexiva, condizente com a filosofia hermenêutico-dialética, a qual considera que o discurso desse grupo de participantes está relacionado às narrativas e experiências de cada indivíduo na relação com: (i) os demais; (ii) o contexto imediato da instituição na qual essas narrativas foram produzidas; e, mais amplamente, (iii) o contexto histórico em que estavam todos envolvidos ${ }^{26}$.

Com o propósito de examinar os dados em sua totalidade, a escuta sistemática das narrativas das participantes do estudo foi realizada paralelamente às transcrições das entrevistas. Além do mais, a leitura dos resultados quantitativos e sociodemográficos foi retomada. Durante as primeiras leituras da transcrição das falas, foi iniciado o processo predominantemente hermenêutico de realizar observações quanto às falas recorrentes ou que estavam diretamente ligadas ao objetivo do estudo. A partir dessas observaçóes, o conteúdo textual foi submetido a uma análise lexicográfica, com auxílio do software Interface de $R$ pour Analyses Multidimensionnel de Textes et de Questionnaires (IRAMUTEQ), que gerou o agrupamento das falas em unidades semânticas semelhantes e separou as classes de declaraçôes significativas com uso da Classificação Hierárquica Descendente $(\mathrm{CHD})^{27}$. A partir da descrição textual e estrutural dos fenômenos, uma análise fatorial por correspondência (AFC) geral e específica foi realizada no mesmo software, a fim de identificar o conteúdo das narrativas entre a maior frequência das palavras evocadas dentro das classes emergidas ${ }^{27}$. A seguir, analisaram-se se as unidades semânticas geradas que apresentavam expressividade e relevância para a compreensão da experiência das pacientes. Procedeu-se à nomeação das classes que se formaram com as unidades de sentido, oriundas das falas das participantes, de forma semelhante a uma análise temática em uma análise de conteúdo ${ }^{26}$. Com os temas identificados e caracterizados, passou-se para o processo interpretativo para categorização dos conteúdos e sua posterior análise, também denominada de dialética ${ }^{26}$.

\section{RESULTADOS}

\section{DADOS QUANTITATIVOS}

Este estudo foi composto por 78 mulheres, com idade média de 55,8 $\pm 11,7$ anos. Destas, 42,3\% eram casadas $(\mathrm{p}<0,005), 23,1 \%$, solteiras, e as demais, divorciadas ou viúvas. No item cor, a maioria $(56,4 \%)$ se autodeclarou branca $(\mathrm{p}<0,005)$, seguida pelas participantes pardas $(32,1 \%)$, pretas $(9 \%)$ e amarelas $(2,6 \%)$. O catolicismo foi declarado como principal religião $(48,7 \%)$, com diferença significante $(\mathrm{p}<0,005)$, seguido pela religião evangélica $(28,2 \%)$ e pelo espiritismo (10,3\%). A maioria das pacientes era de baixa renda, vivendo com uma quantia mensal entre um $(\mathrm{p}<0,005)$ e três salários-mínimos $(91 \%)$, e tinha baixo grau de escolaridade $(\mathrm{p}<0,005)$, com predomínio de ensino fundamental incompleto $(42,3 \%)$. Além disso, mais da metade das participantes $(51,3 \%)$ declararam que seus núcleos familiares eram compostos por pelo menos três pessoas $(\mathrm{p}<0,005)$ (Tabela 1). Tais resultados são relatados de acordo com as diretrizes estabelecidas pelo Strengthening the Reporting of Observational Studies in Epidemiology (STROBE).

Pode-se observar, na Tabela 2 , que cerca de $72,4 \%$ das participantes deste estudo tinham idade acima de 50 anos, e $50 \%$ da amostra fizeram uso de contraceptivos orais durante a vida. Mais de 59\% eram alcoolistas crônicas, aproximadamente $28,2 \%$ eram fumantes ativas e passivas, e cerca de $35 \%$ possuíam histórico de câncer de mama na família.

Quanto às características clínicas, hormonais e terapêuticas das participantes do estudo, o tempo de diagnóstico foi em média de $2,7 \pm 2,4$ anos e essas mulheres estavam na pós-menopausa $(74,4 \% ; n=58)$; tinham predominância de carcinoma ductal invasivo $(85,9 \%$; $\mathrm{n}=67$ ); apresentavam estadiamento clínico oscilando em proporção equivalente entre os estádios II e III; e estavam majoritariamente em regime de quimioterapia adjuvante $(89,5 \% ; n=69)$.

$\mathrm{Na}$ Tabela 3, observa-se que o nível de esperança das participantes ficou próximo do escore total do instrumento (48 pontos), e que a média dos escores da maioria dos domínios de QV foi mediana (acima de 60 pontos), exceto os domínios capacidade funcional $(28,2 \pm 39,6)$ e saúde mental $(30,8 \pm 43,3)$. 
Tabela 1. Características sociodemográficas das mulheres com câncer de mama participantes do estudo ( $\mathrm{n}=78$ )

\begin{tabular}{|c|c|c|}
\hline Variável & Medida & $\mathrm{X}^{2}$ (N; GL); P valor \\
\hline Idade (anos), média \pm DP & $55,8 \pm 11,7$ & \\
\hline \multicolumn{3}{|l|}{ Estado civil, n (\%) } \\
\hline Casada & $33(42,3)$ & $13,4(76 ; 3) ;<0,0004$ \\
\hline Solteira & $18(23,1)$ & \\
\hline Divorciada & $12(15,4)$ & \\
\hline Viúva & $15(19,2)$ & \\
\hline \multicolumn{3}{|l|}{ Cor, n (\%) } \\
\hline Branca & $44(56,4)$ & $56,1(76 ; 3) ;<0,000$ \\
\hline Preta & $7(9,0)$ & \\
\hline Parda & $25(32,1)$ & \\
\hline Amarela & $2(2,6)$ & \\
\hline \multicolumn{3}{|l|}{ Religião, n (\%) } \\
\hline Católica & $38(48,7)$ & $78,7(76 ; 5) ;<0,000$ \\
\hline Espírita & $8(10,3)$ & \\
\hline Evangélica & $22(28,2)$ & \\
\hline Umbanda & $3(3,8)$ & \\
\hline Outra & $1(1,3)$ & \\
\hline Sem religião & $6(7,7)$ & \\
\hline \multicolumn{3}{|l|}{ Renda (número de SM), n (\%) } \\
\hline$<1$ & $3(5,1)$ & $58,3(76 ; 4) ;<0,000$ \\
\hline 1 a 3 & $71(91)$ & \\
\hline$>3$ & $2(3,8)$ & \\
\hline \multicolumn{3}{|l|}{ Escolaridade, n (\%) } \\
\hline Ensino Fundamental incompleto & $33(42,3)$ & $67,2(76 ; 6) ;<0,000$ \\
\hline Ensino Fundamental completo & $17(21,8)$ & \\
\hline Ensino Médio incompleto & $6(7,7)$ & \\
\hline Ensino Médio completo & $13(16,7)$ & \\
\hline Ensino Superior incompleto & $3(3,8)$ & \\
\hline Ensino Superior completo & $5(6,4)$ & \\
\hline Pós-graduação & $1(1,3)$ & \\
\hline \multicolumn{3}{|c|}{ Número de pessoas na moradia, n (\%) } \\
\hline 1 & $8(10,3)$ & $58,3(76 ; 4) ;<0,000$ \\
\hline 2 & $19(24,4)$ & \\
\hline 3 & $40(51,3)$ & \\
\hline 4 & $10(12,8)$ & \\
\hline$>4$ & $1(1,3)$ & \\
\hline
\end{tabular}

Legendas: $\mathrm{SM}$ = salário-mínimo mensal previsto para a populaçăo brasileira; $\mathrm{DP}=$ desvio-padrāo; $\mathrm{X}^{2}$ = qui-quadrado; $\mathrm{N}=$ populaçáo; $\mathrm{GL}=$ grau de liberdade.

A Tabela 4 mostra que não houve diferença na percepção das participantes sobre sua QVRS ( $p>0,005)$ nem mudança de sua saúde percebida ao longo do tempo de diagnóstico ( $p>0,005)$, mas houve redução da esperança de cura daquelas com diagnóstico acima de 5 $\operatorname{anos}(\mathrm{p}<0,005)$.

\section{DADOS QUALITATIVOS}

Obteve-se a narrativa de dez mulheres com câncer de mama, com idade média de $58 \pm 8$ anos e tempo de diagnóstico de $3 \pm 2$ anos em média. Todas tinham carcinoma ductal invasivo, apresentavam estadiamento clínico de estádio I $(10 \%, \mathrm{n}=1)$, oscilando em proporção 
Tabela 2. Fatores de risco para câncer de mama declarados pelas participantes do estudo $(n=78)$

\begin{tabular}{ll}
\multicolumn{1}{c}{ Fatores de risco } & Medida \\
\hline Idade > 50 anos, n (\%) & $55(72,4)$ \\
\hline Endócrinos e história reprodutiva, $\mathbf{n}(\%)$ & \\
Nuliparidade & $9(11,5)$ \\
Uso de contraceptivos orais & $39(50)$ \\
Terapia de reposição hormonal & $17(21,8)$ \\
\hline Comportamentais e ambientais, n (\%) \\
Uso de bebida alcoólica & $47(60,3)$ \\
Tabagismo & $22(28,2)$ \\
Convivência com fumante & $22(28,2)$ \\
\hline Genéticos e hereditários, $\mathbf{n}(\%)$ & \\
Câncer de mama na família & $27(34,6)$ \\
\hline
\end{tabular}

Tabela 3. Média dos escores e confiabilidade (alfa de Cronbach) dos domínios SF-36-RAND e da Escala de Esperança de Herth das participantes do estudo $(n=78)$

\begin{tabular}{lcc}
\hline Variável & Média \pm DP & $\begin{array}{c}\text { Alfa de } \\
\text { Cronbach }\end{array}$ \\
\hline AF & $68,2 \pm 21,5$ & 0,9 \\
CF & $28,2 \pm 39,6$ & 0,8 \\
EGS & $59,9 \pm 15,5$ & 0,6 \\
Dor & $60,6 \pm 25,2$ & 0,6 \\
VT & $63,3 \pm 17,5$ & 0,7 \\
AE & $64,2 \pm 18$ & 0,7 \\
AS & $65,9 \pm 23,8$ & 0,6 \\
SM & $30,8 \pm 43,3$ & 0,8 \\
HHI & $40,2 \pm 5,2$ & 0,9 \\
\hline
\end{tabular}

Legendas: $\mathrm{DP}=$ desvio-padrăo; $\mathrm{AF}=$ aspectos físicos; $\mathrm{CF}=$ capacidade funcional; $\mathrm{EGS}=$ estado geral de saúde; $\mathrm{VT}=$ vitalidade; $\mathrm{AE}=$ aspectos emocionais; $\mathrm{AS}=$ aspectos sociais; $\mathrm{SM}=$ saúde mental; HHI = Escala de Esperança de Herth (do inglês, Herth Hope Index). equivalente entre os estádios II $(30 \%, \mathrm{n}=3)$, III $(30 \%, \mathrm{n}=3)$ e IV $(30 \%, n=3)$, e estavam em regime de quimioterapia adjuvante $(70 \% ; \mathrm{n}=7)$ e neoadjuvante $(30 \%, \mathrm{n}=3)$.

O corpus geral foi constituído por dez textos, separados em 243 segmentos de texto (ST), com aproveitamento de 209 ST (86,01\%). Emergiram 7.756 ocorrências (palavras, formas ou vocábulos), das quais 3.918 foram palavras distintas e 773 tiveram ocorrência única. O conteúdo analisado foi categorizado e gerou as classes 1 , com 71 ST $(33,97 \%) ; 2$ com 59 ST $(28,23 \%)$; e 3 com 79 ST $(37,80 \%)$.

$\mathrm{Na}$ Figura 1, observam-se as três classes se dividindo nas ramificaçōes A e B do corpus total em análise. O subcorpus A, Enfrentamento, é composto pela Classe 1 - Esperança-, que se refere ao comportamento emocional da participante frente à doença, incluindo, principalmente, busca de força na espiritualidade e nas pessoas em seu entorno. Já o subcorpus $\mathrm{B}$, Aspectos Biológicos, é representado pela Classe 2, denominada $A$ doença. O discurso correspondente à Classe 3 - Assistência Clínica - representa a rede de assistência disponibilizada para o cuidado (Figura 1).

Pode-se identificar as palavras associadas à Classe 1 na Figura 1. Os fragmentos a seguir justificam a dimensão dessa classe:

\begin{abstract}
Então, assim, eu tô super bem com isso. Cada dia fico mais feliz mesmo. Eu não sinto nada. Sou muito feliz, sempre pra cima. Orei para Deus. Deus sempre me deu força, agora. E eu pedi, agora força além do normal. E estou indo bem. Eu continuei e tenho esperança (Flor de Lis).
\end{abstract}

A gente tem que falar graças a Deus, sabe? Graças a Deus. Sem ele não temos força e podemos afundar na tristeza, no medo. Entáo assim. A gente

Tabela 4. Comparação entre as médias dos escores de qualidade de vida relacionada à saúde, de esperança e mudança na percepção de saúde das mulheres com câncer de mama de acordo com o tempo de diagnóstico em anos

\begin{tabular}{lcccc}
\hline Variáveis & $<\mathbf{1}$ ano $(\mathbf{n = 1 5})$ & $\mathbf{1}$ a $\mathbf{3}$ anos $(\mathbf{n}=\mathbf{3 6})$ & $\mathbf{5}$ anos $(\mathbf{n}=\mathbf{2 7})$ & $\mathbf{F}(\mathbf{G L}) ; \mathbf{P}$ valor \\
\hline AF & $78,0 \pm 18,0$ & $65,0 \pm 20,1$ & $67,1 \pm 23,3$ & $2,06(2) ; 0,134$ \\
CF & $28,3 \pm 40,0$ & $25,0 \pm 39,2$ & $32,4 \pm 40,9$ & $0,26(2) ; 0,768$ \\
SM & $22,2 \pm 41,2$ & $30,5 \pm 43,2$ & $30,8 \pm 43,3$ & $0,47(2) ; 0,627$ \\
VT & $56,7 \pm 12,5$ & $65,7 \pm 18,4$ & $63,7 \pm 18,2$ & $1,45(2) ; 0,242$ \\
AE & $60,0 \pm 17,2$ & $66,4 \pm 19,8$ & $62,8 \pm 16,0$ & $0,74(2) ; 0,481$ \\
AS & $56,9 \pm 22,3$ & $71,3 \pm 21,6$ & $63,6 \pm 26,1$ & $2,21(2) ; 0,116$ \\
Dor & $55,9 \pm 23,7$ & $60,9 \pm 26,1$ & $60,6 \pm 25,2$ & $0,35(2) ; 0,704$ \\
EGS & $56,3 \pm 14,0$ & $59,2 \pm 16,6$ & $42,0 \pm 15,0$ & $0,89(2) ; 0,412$ \\
HHI & $42,0 \pm 5,6^{A}$ & $41,6 \pm 4,6$ & $37,0 \pm 4,4^{\mathrm{B}}$ & $8,49(2) ; 0,000$ \\
MS & $43,3 \pm 17,6$ & $55,6 \pm 24,4$ & $53,2 \pm 22,2$ & $1,76(2) ; 0,180$ \\
\hline
\end{tabular}

Legendas: $\mathrm{AF}=$ aspectos físicos; $\mathrm{CF}=$ capacidade funcional; $\mathrm{SM}=$ saúde mental; $\mathrm{VT}=$ vitalidade; $\mathrm{AE}=$ aspectos emocionais; $\mathrm{AS}=$ aspectos sociais; $\mathrm{EGS}=$ estado geral de saúde; HHI = Escala de Esperança de Herth (do inglês, Herth Hope Index); MS = mudança na saúde; F = teste F; GL = grau de liberdade; a e b = médias com índices diferentes nas linhas apresentam $\mathrm{p}<0,05$. 


\begin{tabular}{|c|c|c|c|c|c|c|c|c|}
\hline \multicolumn{6}{|c|}{ (A) Enfrentamento } & \multicolumn{3}{|c|}{ (B) Aspectos biológicos } \\
\hline \multicolumn{3}{|c|}{$\begin{array}{l}\text { Classe } 1 \text { - Esperança (34\%) } \\
\text { - Tempo de diagnóstico > 3anos (40\%): } \\
\left.\text { (25 ST; } \mathrm{X}^{2} 18,3\right) \\
\text { - Idade } 40-50 \text { anos }(n=3):\left(25 S T ; X^{2}=18,3\right)\end{array}$} & \multicolumn{3}{|c|}{$\begin{array}{l}\text { Classe } 2 \text { - A doença (28\%) } \\
\text { - Tempo de diagnóstico de } 1 \text { a } 2 \text { anos } \\
\left.\text { (40\%): (10 ST; } \mathrm{X}^{2}=5,2\right) \\
\text { - Idade 51-69 anos }(\mathrm{n}=4):\left(10 \mathrm{ST} ; \mathrm{X}^{2}=5,2\right)\end{array}$} & \multicolumn{3}{|c|}{$\begin{array}{l}\text { Classe } 3 \text { - Assistência clínica (38\%) } \\
\text { - Tempo de diagnóstico < } 1 \text { ano }(20 \%): \\
\left.\text { (52 ST; } X^{2}=11,7\right) \\
\text { - Idade }>70 \text { anos }(n=2):\left(91 \mathrm{ST} ; X^{2}=20,2\right)\end{array}$} \\
\hline Palavra & $f(\%)$ & $\mathrm{X}^{2} ; \mathrm{P}$ valor & Palavra & $f(\%)$ & $\mathrm{X}^{2} ; \mathrm{P}$ valor & Palavra & $f(\%)$ & $\mathrm{X}^{2} ; \mathrm{P}$ valor \\
\hline Deus & $23(87)$ & 32,$4 ;<0,01$ & Receber & $8(100)$ & 21,$2 ;<0,01$ & Médico & $35(83)$ & 36,$3 ;<0,01$ \\
\hline Bem & $28(79)$ & 28,$7 ;<0,01$ & Medo & $8(100)$ & 21,$2 ;<0,01$ & Aí & $73(62)$ & 27,$1 ;<0,01$ \\
\hline Gente & $39(67)$ & 22,$9 ;<0,01$ & Mãe & $8(100)$ & 21,$2 ;<0,01$ & Lá & $24(79)$ & 19,$7 ;<0,01$ \\
\hline Força & $11(100)$ & 22,$6 ;<0,01$ & Doença & $8(100)$ & 21,$2 ;<0,01$ & Doutor & $9(100)$ & 15,$5 ;<0,01$ \\
\hline Graça & $13(77)$ & 11,$4 ;<0,01$ & Câncer & $14(79)$ & 18,$8 ;<0,01$ & Falar & $60(58)$ & 15,$1 ;<0,01$ \\
\hline Esperança & $8(88)$ & 10,$6 ;<0,01$ & Parecer & $8(88)$ & 14,$4 ;<0,01$ & Exame & $20(75)$ & 13,$0 ;<0,01$ \\
\hline Cabeça & $10(80)$ & 9,$9 ;<0,01$ & Ano & $14(72)$ & 13,$8 ;<0,01$ & Voltar & $10(90)$ & 12,$2 ;<0,01$ \\
\hline Mundo & $7(86)$ & 8,$7 ;<0,01$ & Morrer & $5(100)$ & 13,$0 ;<0,01$ & Mastologista & $6(100)$ & 10,$2 ;<0,01$ \\
\hline Sentir & $15(77)$ & 7,$7 ;<0,01$ & Notícia & $7(86)$ & 11,$8 ;<0,01$ & Mandar & $6(100)$ & 10,$2 ;<0,01$ \\
\hline Bom & $16(63)$ & 6,$3 ;<0,01$ & Quimio & $7(86)$ & 11,$8 ;<0,01$ & olhar & $11(82)$ & 9,$6 ;<0,01$ \\
\hline Confiar & $3(62)$ & 5,$9 ;<0,01$ & Falecer & $4(100)$ & 10,$4 ;<0,01$ & Tirar & $13(77)$ & 9,$0 ;<0,01$ \\
\hline Feliz & $3(100)$ & 5,$9 ;<0,01$ & Pai & $4(100)$ & 10,$4 ;<0,01$ & Mama & $8(88)$ & 8,$8 ;<0,01$ \\
\hline Rezar & $3(100)$ & 5,$9 ;<0,01$ & Ruim & $6(84)$ & 9,$3 ;<0,01$ & Municipal & $5(100)$ & 8,$4 ;<0,01$ \\
\hline Acalmar & $3(100)$ & 5,$9 ;<0,01$ & Irmão & $6(84)$ & 9,$3 ;<0,01$ & Inteira & $5(100)$ & 8,$4 ;<0,01$ \\
\hline Continuar & $5(80)$ & 4,$9 ;<0,01$ & Nunca & $6(84)$ & 9,$3 ;<0,01$ & Chorar & $10(80)$ & 8,$0 ;<0,01$ \\
\hline Trabalhar & $7(72)$ & 4,$5 ;<0,01$ & Dormir & $3(100)$ & 7,$8 ;<0,01$ & Mamografia & $14(72)$ & 7,$2 ;<0,01$ \\
\hline
\end{tabular}

Figura 1. Classificação hierárquica descendente das palavras evocadas nas classes pelas pacientes, de acordo com a faixa etária e o tempo de diagnóstico

tem esperança que tudo vai dar certo. E Deus no comando de tudo (Jasmin).

A Classe 2, identificada como a dimensão da doença, enfatiza a evocação das palavras que podem ser identificadas tanto na Figura 1 quanto nos fragmentos a seguir:

Porque, poxa, uma pessoa que faz mamografia, que não bebe, não fuma, não faz nada e de repente tem câncer? Eu fiquei muito revoltada. Mas depois é diferente, né? Só que meu câncer, ele não saiu da mama, não passou no pulmão, ficou localizado (Margarida).

Minha mãe já tinha tido. Ela faleceu com câncer de mama, entáo eu sempre fazia mamografia e quando recebi a notícia eu recebi normal. Vou tratar. Eu já fazia mamografia por causa da minha mãe. Acompanhei minha mãe só no começo do tratamento, pois depois ela largou e ficou por conta própria. Só começou a tratar depois, quase no fim da vida. Tinha mais de dez anos que ele estava com ela, e ela durou muito (Tulipa).

Na Figura 1, é possível identificar as palavras associadas à dimensão assistência à saúde listadas na Classe 3. Os fragmentos a seguir, retirados de algumas das narrativas, exemplificam essa categoria:

Voltei pro médico, conversei com ele. Ele achou um absurdo. Mas depois a gente discutiu. Aí pediu outra mamografia. Só que a outra mamografia já mostrou o caroço muito maior. Aí foi onde comecei meu tratamento na minha cidade. Fui procurar um oncologista, fiz todos os exames, tirei um pedacinho 
pra biópsia, aí deu. Daí fui transferida pra cá (Violeta).

Eu percebi um carocinho. Assim, eu sempre faço exame de toque tá? E eu percebi um carocinho de nada. Eu já falei: ó, tem um carocinho aqui. Fui correndo no postinho perto da minha casa e disse: estou com um carocinho aqui e quero falar com médico urgente! Pra ver se aí eles me encaixariam na mesma hora (Orquídea).

\section{DISCUSSÃO}

Os resultados do estudo que investigou mulheres com câncer de mama confirmam a hipótese de que a doença e o seu tratamento alteram a percepção de QVRS das pacientes e mantêm a esperança de melhora de maneira geral. No entanto, segundo os depoimentos, após cinco anos da doença, o sentimento de medo de recidiva é uma realidade. Com o início dos primeiros sintomas, diagnóstico, tratamento e acompanhamento, mulheres que lutam contra o câncer de mama têm suas vidas modificadas e enfrentam vários entraves pessoais, familiares e sociais.

O questionário sociodemográfico possibilitou identificar variáveis relevantes das pacientes, tais como idade, estado civil, cor, religião, escolaridade e número de residentes do núcleo familiar. A média de idade das participantes está em concordância com os achados do Instituto Nacional de Câncer José Alencar Gomes da Silva (INCA), que relata aumento progressivo do câncer de mama após os 35 anos e maior incidência após os $50 \mathrm{anos}^{28}$. Tais dados são convergentes com os padróes analisados em estudo brasileiro recente, que afirma que as características sociodemográficas, que coincidem com as deste estudo, são verdadeiros marcadores de vulnerabilidade social ${ }^{29}$. Esse mesmo estudo afirma que baixo grau de escolaridade, baixa renda e núcleos familiares são por si só determinantes negativos, pois refletem no acesso aos serviços, no diagnóstico tardio e nas condiçôes inferiores de recuperaçấo (suporte familiar e social) durante o tratamento da doença ${ }^{29}$, o que demonstra a necessidade de estratégias para superar ou, ao menos, minimizar tais efeitos ${ }^{30}$.

Este estudo demonstrou ainda a manutençáo de alguns fatores de risco das pacientes. De acordo com Balekouzou et al. ${ }^{31}$, a utilização de contraceptivos orais e a ingesta de bebidas alcoólicas estão associadas ao desenvolvimento de câncer de mama e são categorizadas como fatores de risco modificáveis, devendo ser abordadas durante a prevenção e o tratamento das patologias mamárias. De outro lado, tem-se a presença de histórico familiar, que se enquadra em fator de risco não modificável, por ter associação genética e alta probabilidade de expressão dos oncogenes BRCA1 e BRCA. Além de desencadearem o surgimento do câncer mamário, esses genes estão associados aos subtipos mais agressivos $^{32}$.

Por meio da Escala de Esperança de Hert, pode-se identificar que a maioria das pacientes (cerca de 90\%) tinha perspectivas positivas quanto ao tratamento e ao prognóstico de sua condição em saúde, mas as narrativas revelaram uma dicotomia entre medo e esperança. Muitas das perspectivas positivas tiveram associação direta com a religiosidade e crenças, que possibilitaram a cada paciente apegar-se a uma fé não mensurável, conforme é possível visualizar nos seguintes trechos: "e também a fé em Deus, procurando sempre buscar em Deus assim força né" e "com a fé em Deus, eu procuro, sempre busquei forças em Deus, que é assim o que tem me sustentado até hoje”.

Apesar das limitaçóes impostas pela doença e das modificaçôes expressivas no cotidiano, grande parte das pacientes manteve suas atividades mínimas, como cuidar da casa, cuidar da família e interagir com os amigos. Vale ressaltar que houve concordância entre as pacientes de que o suporte familiar, os círculos de convívio e a fé são ferramentas fundamentais para nutrir a esperança de cura e de superação da doença. Entretanto, houve relato de alteração na relação marital, com queixa de que o relacionamento íntimo ficou estremecido e que a retirada da mama mexeu com a autoestima da maioria das participantes, o que coincide com os relatos de Bucher-Maluschke et al..$^{33}$ e de Fanakidou et al. ${ }^{3}$, ao concluírem que a reconstrução mamária não é uma panaceia para pacientes com câncer de mama na melhoria da sua QVRS.

$\mathrm{O}$ medo da progressão da doença esteve presente em proporçóes diferentes durante o processo de adoecimento de cada paciente. Tal sentimento mostrou-se expressivo e em demasia nos momentos de diagnóstico, piora dos sintomas e efeitos colaterais da quimioterapia, como enjoos, fraqueza e queda de cabelo. A imagem do corpo fragilizado ocasionava medo e minava as esperanças de superação, como se pode visualizar nos seguintes trechos: "foi muito assustador pra mim, porque de repente eu trabalhava, eu tinha uma vida assim normal, e de repente veio aquela, né, aquele diagnóstico que me assustou muito, muito, eu fiquei sem chão" e "eu achava que ia morrer a qualquer instante e aquilo vivia na minha mente... $\mathrm{E}$ eu perdia às vezes o sono, me via pensando, então é muito angustiante mesmo".

Contudo, o suporte das redes de apoio a essas pacientes exerceu papeis fundamentais de amparo. Algumas desenvolveram depressão, outras, crises de ansiedade, outras barganharam o tratamento, mas a maioria enfrentou esses momentos de maneira positiva: "Não é fácil, mas buscando em Deus, Deus dá essa paz que a gente 
precisa. A gente tem que se amar, se respeitar em primeiro lugar e pensar que não somos inferiores a ninguém por tá passando por essa situação".

O somatório desses fatores é expressivo ao analisarmos os escores dos domínios de QV dessas pacientes. Grande parte das mulheres manteve, ou tentou manter, seu cotidiano inalterado, suas atividades laborais intactas e suas inter-relaçóes pessoais ativas. A experiência do adoecimento na QV é extremamente expressiva na mudança de perspectiva do "hoje" em relação ao "ontem" e ao "amanhâ", promovendo o exercício de planos e atividades postergadas no cotidiano pela perspectiva do bom e do novo, inovando-se, reconstruindo-se e ressignificando-se. Essas características são expressas claramente pelas mulheres da pesquisa, tal como a paciente a seguir: "às vezes, a gente leva a vida muito no automático e não é bem isso. Acho que você tem que amenizar as coisas. O dia do amanhã a gente não sabe, então a gente tem que viver o hoje o melhor possível".

A adoçáo de uma metodologia mista incidiu mais luz sobre os resultados e possibilitou a confirmação de que o diagnóstico de câncer provoca impacto negativo emocional no paciente e nos seus familiares, porque culturalmente ele é associado à morte, à finitude ${ }^{33}$, que é um dos sentimentos mais primitivos que uma pessoa pode experenciar ${ }^{34}$ pela perda de esperança de cura $^{35}$. Ademais, um dos aspectos da fenomenologia é o da compreensão do fenômeno pela percepção de quem o vivencia. Foi possível observar a dicotomia de sentimentos positivos e negativos justamente porque cada pessoa tem seu processo de adoecimento biológico atrelado a um contexto sociocultural, o chamado relativismo cultural ${ }^{36}$. Ressalta-se que o uso de análise quantitativa e das narrativas amparadas no referencial teórico da fenomenologia possibilitou um processo cíclico constante de ir e vir com as análises, proporcionado pelo círculo hermenêutico-dialético ${ }^{26}$, que permitiu finalizar essa investigação detectando o significado, para essas mulheres, dos sentimentos de medo sem perder a esperança e com uma percepção moderada de QV.

O SF-36 é um instrumento genérico de QV e permite comparaçôes entre pacientes com diferentes condiçôes crônicas. Os instrumentos genéricos podem, no entanto, ser indiferentes às mudanças em condiçōes específicas, como no caso do câncer de mama, pois o foco náo é a doença em particular. Essa limitação metodológica é inerente aos instrumentos genéricos. Nesse sentido, o uso da metodologia qualitativa no presente estudo foi importante para confirmação dos resultados obtidos com esse questionário.

As implicaçóes específicas para a experiência do diagnóstico e do tratamento das mulheres com câncer de mama são as de que as intervençóes a seguir devem ser implementadas com base nos resultados deste estudo. Primeiro, médicos e coordenadores de serviços de diagnóstico do Sistema Único de Saúde (SUS) devem responder melhor às solicitaçóes de seus pacientes em espera de diagnóstico com rapidez e precisão. Os médicos também devem incorporar uma descrição mais sucinta dos escores dos domínios do SF-36, ajudando o paciente a interpretar sua situaçáo e a si mesmo para além de uma pontuação. As equipes de saúde devem ampliar sua definição de membro da equipe para incluir os pacientes e a família, além de considerar seus pontos de vista na definiçâoo de políticas gerais. Por fim, os coordenadores de saúde devem desenvolver uma rede, talvez pela Internet, de comunicação e discussão de questôes de perda do eu-passado e questôes sobre a dinâmica do tratamento de câncer de mama. As salas de bate-papo para os pacientes devem servir como um bom ponto de partida e podem ser presenciais ou virtuais. Os profissionais de saúde mental que trabalham com essa população devem considerar um trabalho em grupo ou individual na reconstrução de uma narrativa biográfica coerente no decorrer do período que vai do diagnóstico, passa pelo tratamento e termina no acompanhamento. Todas as pacientes deste estudo estavam investindo em um procedimento e tinham esperança de que o medicamento funcionasse para que a vida delas voltasse ao normal. Mas tinham também o fantasma do medo da progressão da doença que, às vezes, surge para amedrontar. As equipes ligadas ao cuidado dessas mulheres devem atuar com tais medidas para, pelo menos, minimizar esta e outras sintomatologias mentais.

\section{CONCLUSÃO}

Concluiu-se que, para as pacientes com câncer de mama participantes deste estudo, o processo de adoecimento atuou como propulsor de experiências que transitaram entre o positivo e o negativo, desencadeando sentimentos diversificados, modificando o cotidiano e promovendo escores reduzidos nos domínios de capacidade funcional e saúde mental de sua QVRS. Identificar e acolher esses resultados são ferramentas imprescindíveis para a prestação de cuidados humanizados em saúde. Tomar posse do fato de que a experiência de adoecimento é individual e o ponto inicial para propor soluçôes e planejar cuidados; é entender e centralizar a paciente como protagonista de sua história, sendo o profissional da saúde apenas facilitador de processos. O reflexo positivo dessa interação se faz presente no enfrentamento da doença pelas pacientes e na proposiçáo de cuidados efetivos pelas equipes de saúde. 


\section{CONTRIBUIÇÕES}

Os autores contribuíram igualmente em todas as etapas do manuscrito e aprovaram a versão final a ser publicada.

\section{AGRADECIMENTOS}

Agradecemos à FAPEMIG pelos recursos para realização deste estudo.

\section{DECLARAÇÃO DE CONFLITO DE INTERESSES}

Nada a declarar.

\section{FONTES DE FINANCIAMENTO}

Bolsa de Iniciação Científica FAPEMIG.

\section{REFERÊNCIAS}

1. World Health Organization [Internet]. Geneva: WHO; c2021. Cancer; [update $2021 \mathrm{Mar} 3$; cited 2020 June 2]. Available from: https://www.who.int/news-room/ fact-sheets/detail/cancer

2. Bray F, Ferlay J, Soerjomataram I, et al. Global cancer statistics 2018: GLOBOCAN estimates of incidence and mortality worldwide for 36 cancers in 185 countries. CA Cancer J Clin. 2018;68(6):394-424. doi: https://doi. org/10.3322/caac. 21492

3. Fanakidou I, Zyga S, Alikari V, et al. Mental health, loneliness, and illness perception outcomes in quality of life among young breast cancer patients after mastectomy: the role of breast reconstruction. Qual Life Res. 2018;27(2):539-43. doi: https://doi.org/10.1007/ s11136-017-1735-x

4. Cobo-Cuenca AI, Martín-Espinosa NM, RodríguezBorrego MA, et al. Determinants of satisfaction with life and self-esteem in women with breast cancer. Qual Life Res. 2019;28(2):379-87. doi: https://doi.org/10.1007/ s11136-018-2017-y

5. Wong CCY, Pan-Weisz BM, Pan-Weisz TM, et al. Selfstigma predicts lower quality of life in Chinese American breast cancer survivors: exploring the mediating role of intrusive thoughts and posttraumatic growth. Qual Life Res. 2019;28(10):2753-60. doi: https://doi.org/10.1007/ s11136-019-02213-w

6. Madrigal R, Bee C. Suspense as an experience of mixed emotions: feelings of hope and fear while watching suspenseful commercials. In: Menon G, Rao AR, editors. Advances in consumer research [Internet]. Proceedings thirty-second Annual Conference of the Association for Consumer Research (ACR); 2004 Oct 7-10; Oregon. Duluth, MN: Association for Consumer Research; c2005 [cited 2019 Dec 12]. Vol. 32. p. 561-567. Available from:
https://www.acrwebsite.org/assets/PDFs/Proceedings / NAACRVol32.pdf

7. Slevin ML, Nichols SE, Downer SM, et al. Emotional support for cancer patients: what do patients really want? Br J Cancer. 1996;74(8):1275-9. doi: https://doi. org/10.1038/bjc.1996.529

8. The WHOQOL Group. The World Health Organization Quality of Life assessment (WHOQOL): position paper from the World Health Organization. Soc Sci Med. 1995;41(10):1403-9. doi: https://doi.org/10.1016/02779536(95)00112-k

9. Jafari N, Farajzadegan Z, Zamani A, et al. Spiritual well-being and quality of life in Iranian women with breast cancer undergoing radiation therapy. Support Care Cancer. 2013;21(5):1219-25. doi: https://doi. org/10.1007/s00520-012-1650-1

10. Huber M, Knottnerus JA, Green L, et al. How should we define health? Br J Cancer. 2011;343:d4163. doi: https://doi.org/10.1136/bmj.d4163

11. Alberto J, Joyner B. Hope, optimism, and self-care among Better Breathers Support Group members with chronic obstructive pulmonary disease. Appl Nurs Res. 2008;21(4):212-7. doi: https://doi.org/10.1016/j. apnr.2006.12.005

12. Evangelista LS, Doering LV, Dracup K, et al. Hope, mood states and quality of life in female heart transplant recipients. J Heart Lung Transplant. 2003;22(6):681-6. doi: https://doi.org/10.1016/s1053-2498(02)00652-6

13. Pinto S, Caldeira S, Martins JC. A esperança da pessoa com cancro: estudo em contexto de quimioterapia. Rev Enf Ref. 2012;3(7):23-31. doi: https://doi.org/10.12707/ RIII11148

14. Frankl VE. Man's search for ultimate meaning. New York: Plenum; 1997.

15. Strauss A, Corbin J. Basics of qualitative research. Thousand Oaks, CA: Sage Publications; 1990.

16. Herth K. Abbreviated instrument to measure hope: development and psychometric evaluation. J Adv Nurs. 1992;17(10):1251-9. doi: https://doi. org/10.1111/j.1365-2648.1992.tb01843.x

17. Sartore AC, Grossi SAA. Escala de Esperança de Herth: instrumento adaptado e validado para a língua portuguesa. Rev Esc Enferm USP. 2008;42(2):227-32. doi: https:// doi.org/10.1590/S0080-62342008000200003

18. Ciconelli RM, Ferraz MB, Santos W, et al. Tradução para a língua portuguesa e validação do questionário genérico de avaliaçáo de qualidade de vida SF-36 (Brasil SF-36). Rev Bras Reumatol. 1999;39(3):143-50.

19. Husserl E. A crise da humanidade europeia e a filosofia. 3. ed. Porto Alegre: Edipucrs; 2008.

20. Creswell JW. Investigação qualitativa e projeto de pesquisa: escolhendo entre cinco abordagens. 3. ed. Porto Alegre, RS: Penso; 2014. 
21. Moustakas C. Phenomenological research methods. Thousand Oaks, CA: Sage Publications; 1994.

22. Cheung J, Hocking P. The experience of spousal carers of people with multiple sclerosis. Qual Health Res. 2004;14(2):153-66. doi: https://doi. org/10.1177/1049732303258382

23. Terwee CB, Mokkink LB, Knol DL, et al. Rating the methodological quality in systematic reviews of studies on measurement properties: a scoring system for the COSMIN checklist. Qual Life Res. 2012;21:651-7. doi: https://doi.org/10.1007/s11136-011-9960-1

24. Cronbach LJ. Coefficient alpha and the internal structure of tests. Psychometrika. 1951;16(3):297-334. doi: https://doi.org/10.1007/BF02310555

25. Brown J, Sorrell JH, McClaren J, et al. Waiting for a liver transplant. Qual Health Res. 2006;16(1):119-36. doi: https://doi.org/10.1177/1049732305284011

26. Minayo MCS. O desafio do conhecimento: pesquisa qualitativa em saúde. 4. ed. São Paulo: Hucitec; 1996.

27. Salviati ME, organizadora. Manual do aplicativo Iramuteq (versão 0.7 Alpha 2 e $\mathrm{R}$ versão 3.2.3) [Internet]. Planaltina, DF: Iramuteq; 2017 [acesso 2020 abril 2]. Disponível em: http://www.iramuteq.org/ documentation/fichiers/manual-do-aplicativo-iramuteqpar-maria-elisabeth-salviati.

28. Instituto Nacional de Câncer José Alencar Gomes da Silva [Internet]. Rio de Janeiro: INCA; [data desconhecida]. Tipos de câncer: câncer de mama; [modificado 2021 jul 13; acesso 2020 maio 23]. Disponível em: https://www. inca.gov.br/tipos-de-cancer/cancer-de-mama

29. Cabral ALLV, Giatti L, Casale C, et al. Vulnerabilidade social e câncer de mama: diferenciais no intervalo entre o diagnóstico e o tratamento em mulheres de diferentes perfis sociodemográficos. Ciên Saúde Colet. 2019;24(2):613-622. doi: https://doi.org/10.1590/141381232018242.31672016

30. Easley J. Motivations for cancer history disclosure among young adult cancer survivors. J Cancer Surviv. 2019;13(3):447-58. doi: https://doi.org/10.1007/ s11764-019-00766-w

31. Balekouzou A, Yin P, Pamatika CM, et al. Reproductive risk factors associated with breast cancer in women in Bangui: a case-control study. BMC Womens Health. 2017;17(1):14. doi: https://doi.org/10.1186/s12905017-0368-0

32. Kemp Z, Turnbull A, Yost S, et al. Evaluation of cancerbased criteria for use in mainstream BRCA1 and BRCA2 genetic testing in patients with breast cancer. JAMA Netw Open. 2019;2(5):e194428. doi: https://doi.org/10.1001/ jamanetworkopen.2019.4428

33. Bucher-Maluschke JSNF, Fialho RBM, Pedroso JS, et al. Dinâmica familiar no contexto do paciente oncológico. Rev NUFEN [Internet]. 2014 [acesso 2020 maio 23];6(1):87-108. Disponível em: http://pepsic.bvsalud. org/pdf/rnufen/v6n1/a05.pdf
34. Friis LS, Elverdam B, Schmidt KG. The patient's perspective: a qualitative study of acute myeloid leukaemia patients' need for information and their information-seeking behaviour. Support Care Cancer. 2003;11(3):162-170. doi: https://doi.org/10.1007/ s00520-002-0424-6

35. Kleinman AM, Eisenberg L, Good B. Culture, illness, and care: clinical lessons from anthropologic and crosscultural research. Ann Intern Med. 1978;88(2):251-8. doi: https://doi.org/10.7326/0003-4819-88-2-251

36. Langdon EJ, Wiik FB. Antropologia, saúde e doença: uma introduçáo ao conceito de cultura aplicado às ciências da saúde. Rev Lat-Am Enferm. 2010;18(3). doi: https://doi.org/10.1590/S0104-11692010000300023

Recebido em 18/8/2020 Aprovado em 11/2/2021 